\title{
A platform for high-repetition-rate laser experiments on the Large Plasma Device
}

\author{
D. B. Schaeffer, L. R. Hofer, E. N. Knall, P. V. Heuer, C. G. Constantin, and C. Niemann \\ Department of Physics and Astronomy, University of California - Los Angeles, Los Angeles, CA 90095, USA \\ (Received 1 December 2017; revised 6 February 2018; accepted 7 March 2018)
}

\begin{abstract}
We present a new experimental platform for studying laboratory astrophysics that combines a high-intensity, highrepetition-rate laser with the Large Plasma Device at the University of California, Los Angeles. To demonstrate the utility of this platform, we show the first results of volumetric, highly repeatable magnetic field and electrostatic potential measurements, along with derived quantities of electric field, charge density and current density, of the interaction between a super-Alfvénic laser-produced plasma and an ambient, magnetized plasma.
\end{abstract}

Keywords: high energy density physics; plasmas astrophysics

\section{Introduction}

Large laser facilities, such as the National Ignition Facility at Lawrence Livermore National Laboratory, are often at the forefront of laser-driven laboratory astrophysics ${ }^{[1,2]}$, in which experiments seek to model astrophysical systems by scaling key dimensionless variables to the laboratory ${ }^{[3,4]}$. An important subset of these experiments is concerned with the interaction of one or more energetic plasmas with an ambient plasma, such as in studies of collisionless shocks ${ }^{[5]}$, magnetic reconnection ${ }^{[6]}$, mini-magnetospheres ${ }^{[7]}$, or selfgenerated magnetic fields ${ }^{[8]}$. However, in regard to such experiments, these facilities suffer from two main drawbacks: an inherently limited shot rate (of order ten or less for a given experimental campaign), and the necessity to dynamically create ambient plasmas with lasers. The low number of shots yields small datasets and reduces the number of parameters that can be explored, while laser-driven ambient plasmas are difficult to control and diagnose.

The Large Plasma Device (LAPD) at the University of California, Los Angeles (UCLA) addresses some of these shortcomings by providing a high-repetition-rate, highly repeatable, long-lived and well-diagnosed ambient plasma. Previous experiments on the LAPD have utilized commercially available, high-repetition-rate lasers to study the magnetic ${ }^{[9-12]}$ and electric fields ${ }^{[13]}$ involved in the formation of diamagnetic cavities. These experiments demonstrated how large, detailed volumetric datasets could be

Correspondence to: D. B. Schaeffer, University of California, Los Angeles, CA 90095, USA. Email: dschaeffer@physics.ucla.edu acquired in laser plasmas, but the lasers employed were too low intensity $\left(\sim 10^{10} \mathrm{~W} / \mathrm{cm}^{2}\right)$ and too low energy $(\lesssim 1.5 \mathrm{~J})$ to drive the highly energetic plasmas necessary for laboratory astrophysics.

In this paper, we overview a new experimental platform at UCLA that combines the LAPD with a high-power, highrepetition-rate laser that is capable of on-target intensities in excess of $10^{14} \mathrm{~W} / \mathrm{cm}^{2}$. This platform allows new 3D volumetric data collection of the interaction between laserdriven plasma plumes and a magnetized ambient plasma. We present the first experimental results using this platform, and discuss its potential application to topics in laboratory astrophysics, including the study of both perpendicular and parallel low-Mach number magnetized shocks, the formation of magnetic instabilities and kinetic-scale magnetospheres.

\section{Experimental platform and setup}

The experiments were carried out at UCLA utilizing a highrepetition-rate laser in the Phoenix Laser Laboratory ${ }^{[14]}$ and the $\mathrm{LAPD}^{[15]}$ operated by the Basic Plasma Science Facility (BaPSF). A schematic of the experimental setup is shown in Figure 1. The LAPD is a $20 \mathrm{~m}$ long, $1 \mathrm{~m}$ diameter cylindrical vacuum vessel that can generate a steady-state ( $\sim 15 \mathrm{~ms}$ ), current-free magnetized ambient plasma at repetition rates up to $1 \mathrm{~Hz}$. Multiple current-carrying coils along the machine provide uniform axial magnetic fields up to $2 \mathrm{kG}$. A BaO-coated $\mathrm{Ni}$ cathode at one end of the machine provides the main 60-cm-diameter ambient plasma, which can be composed of various gases $(\mathrm{H}, \mathrm{D}, \mathrm{He})$. A second 


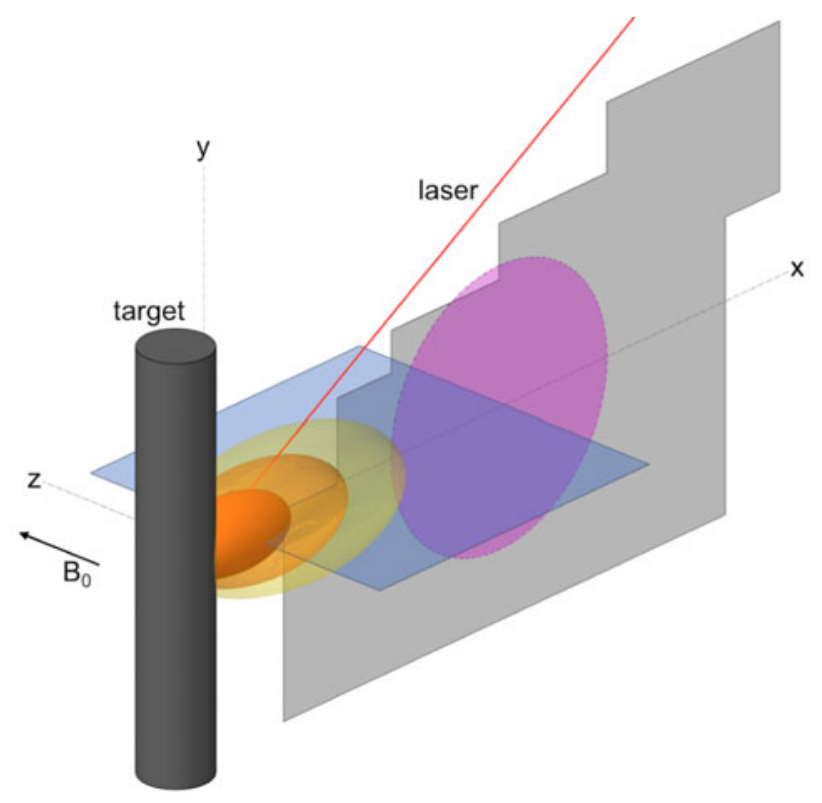

Figure 1. Schematic of the experimental setup in the LAPD. A highrepetition-rate laser hits a plastic target embedded in an ambient magnetized plasma. The target rotates and translates in between laser shots. The resulting interaction between the laser plasma and ambient plasma is scanned with magnetic flux ('bdot') probes and emissive Langmuir probes in two intersecting planes, $x-z$ (blue) and $x-y$ (gray). The location of the high-density ambient plasma at $z=0$ is shown in purple.

$\mathrm{LaB}_{6}$ cathode at the other end of the machine provides a higher-density $20-\mathrm{cm}$-diameter core plasma centered on the main plasma. Consequently, the ambient plasma electron density varies from $n_{e} \approx 5 \times 10^{12} \mathrm{~cm}^{-3}$ at the edge to $n_{e} \approx$ $2 \times 10^{13} \mathrm{~cm}^{-3}$ at the center, with an electron temperature of $\sim 5-10 \mathrm{eV}$ and ion temperature of $\sim 1 \mathrm{eV}$.

The high-repetition-rate laser was originally designed ${ }^{[16,17]}$ at Lawrence Livermore National Laboratory and was recently re-commissioned at UCLA. It can deliver energies of $20 \mathrm{~J}$ at $1053 \mathrm{~nm}$ and variable pulse widths of 8-30 ns (full width at half-maximum, FWHM) in a near-diffractionlimited beam, yielding powers up to $2.5 \mathrm{GW}$, potential intensities in excess of $10^{14} \mathrm{~W} / \mathrm{cm}^{2}$, and repetition rates up to $4 \mathrm{~Hz}$. The output laser energy is stable to within $5 \%$, and the pulse shape, diffraction-limited focus, and beam pointing are stable to within $1 \%$. Additional details on the performance of the laser can be found in Ref. [17].

In the experiments, a high-density polyethylene $\left(\mathrm{C}_{2} \mathrm{H}_{4}\right)$ plastic target, $30.5 \mathrm{~cm}$ long and $38 \mathrm{~mm}$ in diameter, was positioned $30 \mathrm{~cm}$ from the LAPD center axis. The target was mounted on a 2D stepper motor drive synchronized with the laser, which translated and rotated the target in a helical pattern. Each target position was repeated three times and then moved to provide a fresh surface. A single target could thus be used for up to $2 \times 10^{4}$ laser shots. The laser was configured with a 14 ns pulse width (FWHM) and directed through an $f / 10$ lens at an angle of $30^{\circ}$ relative to the target normal, which ablated the target with intensities up to
$I \approx 6 \times 10^{13} \mathrm{~W} \cdot \mathrm{cm}^{-2}$. Due to the low ambient density, the laser had a negligible effect on the ambient plasma over the regions of interest. The target and laser were oriented so that the laser-ablated plasma was directed across the background field. The laser and target were synchronized to the LAPD, and they all operated at a repetition rate of $0.23 \mathrm{~Hz}$ to allow time for the diagnostics to position themselves between shots.

The position of the laser spot on the target defines as $\{x, y, z\}=\{0,0,0\} \mathrm{cm}$, with the background magnetic field directed along $\hat{z}$, the target surface normal directed along $\hat{x}$, and the vertical motion of the target directed along $\hat{y}$. The center of the LAPD is then at $\{x, y\}=\{30,0\} \mathrm{cm}$. Diagnostics was positioned in the planes defined by $z=0$ $(x-y$ plane $)$ or $y=0(x-z$ plane $)$. The firing of the laser defines as $t=0=t_{0}$.

The magnetic field was characterized using $1 \mathrm{~mm}$ diameter, three-axis magnetic flux ('bdot') probes ${ }^{[18]}$. The probes were positioned using a 2D stepper motor drive configured to move in either the $x-z$ or $x-y$ plane. Each position was repeated three times to generate statistics, and a $2 \mathrm{D}$ plane of data was assembled by moving the probe in $5 \mathrm{~mm}$ increments in a grid pattern over successive laser shots. The bdot signals were passed through a $150 \mathrm{MHz}$ differential amplifier and coupled to fast $(1.25 \mathrm{GHz}) 10$-bit digitizers, and then integrated to yield magnetic field.

The electrostatic plasma potential was measured using a resistively heated $\mathrm{CeB}_{6}$ emissive probe ${ }^{[19]}$. For each shot the emissive probe began sampling before the laser fired, which served to measure the ambient plasma potential. This potential was then used as a reference for measurements of the potential taken after the laser fired. Like the bdot probes, the emissive probes were positioned using a $2 \mathrm{D}$ stepper motor drive, and each position was repeated three times and incremented in $5 \mathrm{~mm}$ steps to assemble 2D planes of data.

Images of the interaction of the laser plasma and ambient plasma were acquired with a fast-gate (10 ns) intensified charge-coupled device (ICCD) camera. The camera was positioned either above the target or at the end of the machine to image the $x-z$ and $x-y$ planes, respectively. Data was acquired in $10 \mathrm{~ns}$ increments simultaneously with the measurements of the magnetic field or plasma potential. The camera collected light from broadband plasma selfemission and could be additionally filtered to isolate specific ion charge states. Results from the fast-gate imaging can be found in Ref. [20].

Additionally, swept Langmuir probes were employed to measure similar $x-z$ and $x-y$ planes of plasma electron density and temperature. These measurements were carried out in the absence of the laser plasma, and so provided the initial state of the ambient plasma at $t_{0}$.

\section{Results}

Magnetic field and electrostatic potential measurements were acquired in both the $x-z$ and $x-y$ planes. In the 
Table 1. Typical experimental parameters. The collisional localization is taken with respect to the mean free path $\lambda_{C, H}$ between laser-ablated $\mathrm{C}$ ions and ambient $\mathrm{H}$ ions.

\begin{tabular}{lcc}
\hline Parameter & Symbol & Value \\
\hline System size [cm] & $D$ & 50 \\
Amb. magnetic field [G] & $B_{0}$ & 250 \\
Amb. ele. density $\left[\mathrm{cm}^{-3}\right]$ & $n_{e 0}$ & $1.5 \times 10^{13}$ \\
Amb. ele. temperature [eV] & $T_{e 0}$ & 10 \\
Amb. ion temperature [eV] & $T_{i 0}$ & 1 \\
Amb. Alfvén speed $\left[\mathrm{km} \cdot \mathrm{s}^{-1}\right]$ & $v_{A}$ & 170 \\
Amb. sound speed $\left[\mathrm{km} \cdot \mathrm{s}^{-1}\right]$ & $c_{s}$ & 40 \\
Laser energy [J] & $E_{l}$ & 20 \\
Laser intensity [W/cm $\left.{ }^{2}\right]$ & $I_{l}$ & $6 \times 10^{13}$ \\
Carbon ionization & $Z_{C}$ & 4 \\
Carbon gyroradius $[\mathrm{cm}]$ & $r_{C}$ & 31 \\
Laser-plasma speed $\left[\mathrm{km} \cdot \mathrm{s}^{-1}\right]$ & $v_{0}$ & 250 \\
Magnetic localization & $r_{C} / D$ & 0.6 \\
Collisional localization & $\lambda_{C, H} / D$ & 140 \\
Mach number & $M_{S}=v_{0} / c_{S}$ & 6.3 \\
Alfvénic Mach number & $M_{A}=v_{0} / v_{A}$ & 1.7 \\
\hline
\end{tabular}

$x-z$ plane, the magnetic probe scanned from $x=5.5 \mathrm{~cm}$ to $x=29.5 \mathrm{~cm}$ and $z=-12 \mathrm{~cm}$ to $z=12 \mathrm{~cm}$ over a total of 1875 shots (where each position was repeated three times), while the emissive probe scanned from $x=15 \mathrm{~cm}$ to $x=29 \mathrm{~cm}$ and $z=-8 \mathrm{~cm}$ to $z=12$ using 945 shots. In the $x-y$ plane, in order to avoid hitting the probes with the laser, the probes scanned several smaller regions that were stitched together into a final, roughly triangular region from $x=10 \mathrm{~cm}$ to $x=55 \mathrm{~cm}$ and $y=-15 \mathrm{~cm}$ to $y=18$ over approximately 7000 shots. For all measurements, the background magnetic field was $B_{0}=250 \mathrm{G}$ and directed along $\hat{z}$, and the ambient plasma was composed of $\mathrm{H}^{+1}$ with an initial electron density of $n_{e 0} \approx 1.5 \times 10^{13} \mathrm{~cm}^{-3}$, electron temperature $T_{e 0} \approx 10 \mathrm{eV}$, and ion temperature $T_{i 0} \approx 1 \mathrm{eV}$ (see Figure 2). Typical parameters are listed in Table 1.

The probe measurements were highly repeatable. Each location in a plane was repeated three times. The resulting time-dependent value (magnetic field or electrostatic potential) $F(t)$ was found to be within $5 \%$ of the mean value at that time, i.e., $F(t)=\langle F(t)\rangle \pm 5 \%$. This held for each location that was measured.

Figure 3 combines these $x-z$ and $x-y$ planes into a composite plot at time $t=0.5 \mu \mathrm{s}$ for the $z$-component of the relative magnetic field $\Delta B_{z}=B_{z}-B_{0}$ and the electrostatic potential $\Phi$. Features from each plane are well-aligned, again indicating that the individual measurements are very repeatable over thousands of laser shots. The formation of a fully expelled diamagnetic cavity and leading magnetic compression is clearly visible in $B_{z}$. The cavity has an oblong shape, extending $\sim 20 \mathrm{~cm}$ in both $y$ and $z$ with a maximum extend at this time of $\sim 15 \mathrm{~cm}$ in $x$. At the same time, there is a positive potential of $\Phi \approx 300 \mathrm{~V}$ ahead of the magnetic compression that quickly goes to $\Phi \approx-300 \mathrm{~V}$ at the edge of the magnetic cavity.

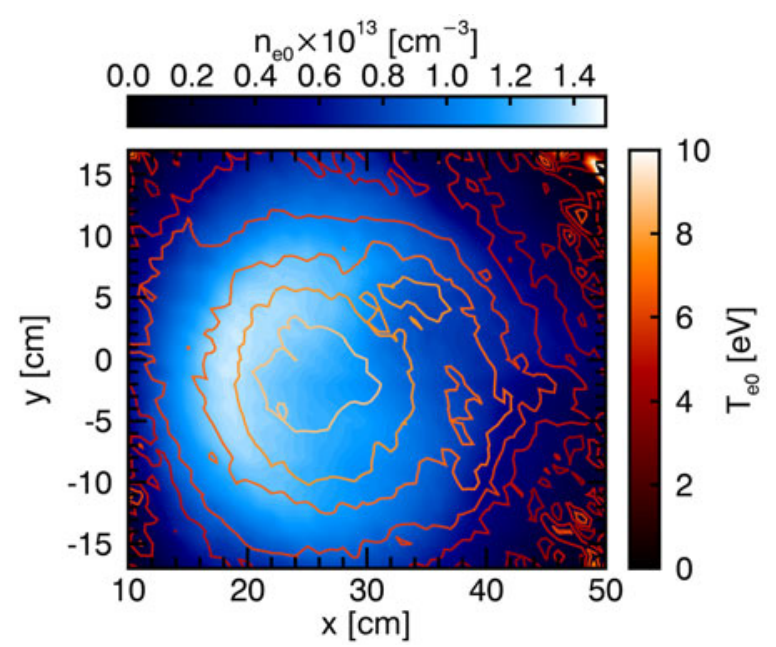

Figure 2. Langmuir probe measurements of the initial electron density $n_{e 0}$ and temperature $T_{e 0}$ in the $x-y$ plane. The target is located at $\{x, y\}=$ $\{0,0\}$.

Figure 4 shows a time series of surface plots of $B_{z}$ in the $x-z$ plane. A fully expelled magnetic cavity is led by a magnetic compression that reaches its peak value $B_{z} / B_{0}=1.7$ at $t \approx 0.5 \mu \mathrm{s}$, at which point it is moving at $v_{0}=250 \mathrm{~km} \cdot \mathrm{s}^{-1}$. This corresponds to an Alfvénic Mach number of $M_{A}=v_{0} / v_{A}=1.8$, where the Alfvén speed $v_{A}=140 \mathrm{~km} \cdot \mathrm{s}^{-1}$ is calculated for the ambient plasma. The cavity itself reaches its maximum size of $17 \mathrm{~cm}$ in $x$ shortly thereafter, and then stagnates at this distance while the compression continues to propagate at $M_{A}=1.5$. Over the next $\sim 1 \mu \mathrm{s}$, the magnetic field diffuses back into the cavity, starting with the cavity edges nearest to the target. This diffusion process results in a long-lived cavity elongated along the background magnetic field, so that the last segment of the cavity to collapse is a narrow strip extending along $z$ at the leading edge. Simultaneously, measurements in the $x-$ $y$ plane indicate that the magnetic compression propagates out to the edge of the LAPD $(x=60 \mathrm{~cm})$ while gyrating upwards $(y>0)$, consistent with the compression being carried by ions.

We note that the cavity still collapses approximately an order of magnitude faster than the classical (Spitzer) or Bohm magnetic diffusion time $\tau_{m}=l^{2} / \eta$, where $l$ is the gradient magnetic scale length on the order of a few $\mathrm{cm}$ and $\eta$ is the diffusivity. At the time of collapse, the laser plasma is mostly confined within the magnetic cavity and has a density $n_{e} \approx 2.5 \times 10^{12}$, temperature $T_{e} \approx 10 \mathrm{eV}$, and average ionization $Z \approx 1.5^{[21]}$. At these parameters, the Bohm diffusivity $\eta_{B}=k_{B} T_{e} / 16 e B_{0} \approx 25 \mathrm{~m}^{2} \cdot \mathrm{s}^{-1}$. The classical diffusivity parallel to the background field $\eta_{S, \|} \approx$ $0.5 \rho_{S} / \mu_{0} \approx 18 \mathrm{~m}^{2} \cdot \mathrm{s}^{-1}$, while the perpendicular diffusivity $\eta_{S, \perp}=\left(v_{e} / \omega_{c e}\right)^{2} \rho_{S} / \mu_{0} \approx 0.03 \mathrm{~m}^{2} \cdot \mathrm{s}^{-1}$, where $\rho_{S}=$ $\pi Z e^{2} m_{e}^{1 / 2} \ln \Lambda /\left(4 \pi \epsilon_{0}\right)^{2}\left(k_{B} T_{e}\right)^{3 / 2}$ is the Spitzer resistivity, $v_{e}$ is the collision frequency, and $\omega_{c e}$ is the gyrofrequency. 
(a)

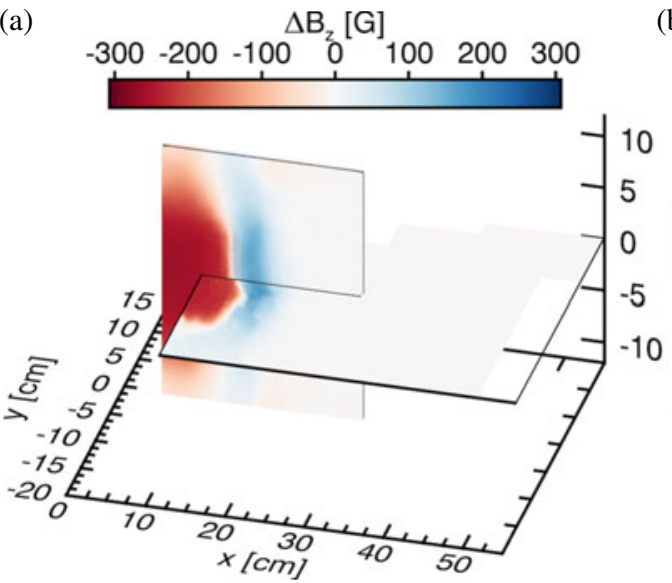

(b)

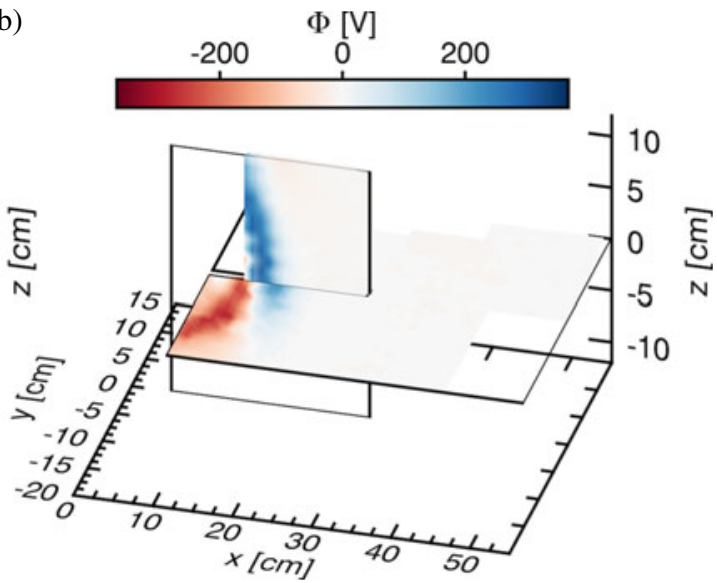

Figure 3. Composite plots of (a) the magnitude of the relative magnetic field $\Delta B_{z}=B_{z}-B_{0}$ and (b) the electrostatic potential $\Phi$ in the $x-z$ and $x-y$ planes at the same time $t=0.5 \mu \mathrm{s}$. Each plane is comprised of thousands of separate laser shots, showing a high degree of reproducibility. The target is located at $\{x, y, z\}=\{0,0,0\}$.
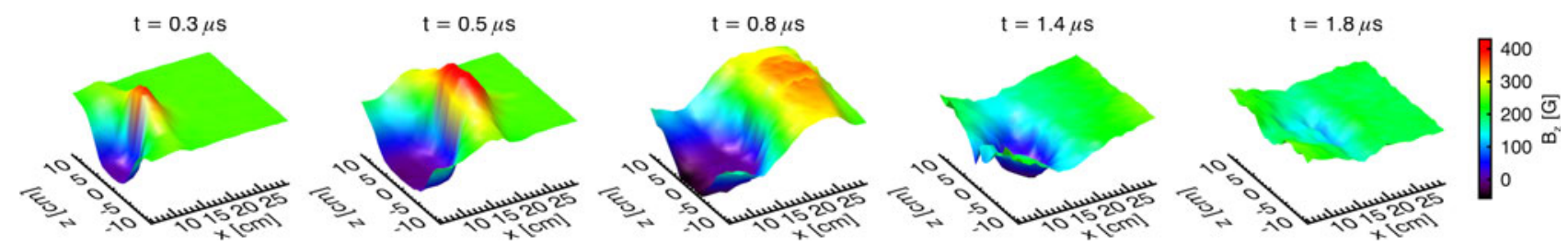

Figure 4. Time series of surface plots of $B_{z}$ in the $x-z$ plane, where the vertical dimension (color) is the magnitude of $B_{z}$. The target is at $\{x, z\}=\{0,0\}$ and the background field $B_{0}=250 \mathrm{G}$ along $\hat{z}$.
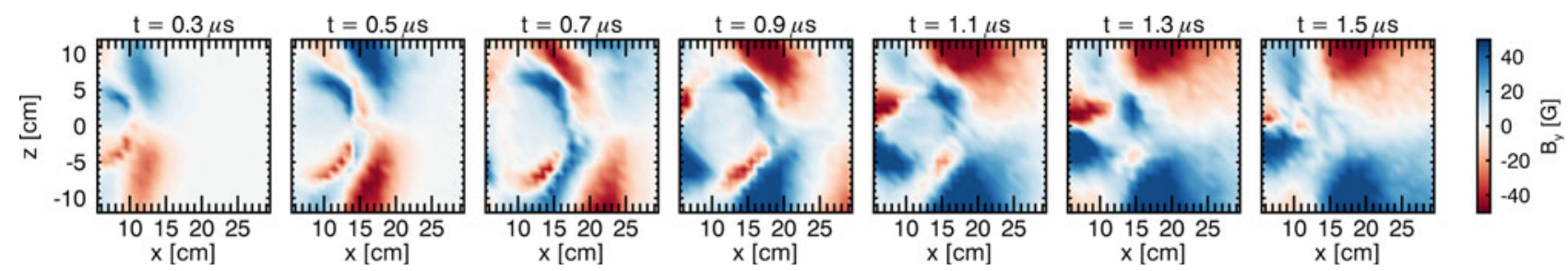

Figure 5. Time series of contour plots of $B_{y}$ in the $x-z$ plane. The target is at $\{x, z\}=\{0,0\}$.

Thus, the fastest (Bohm) diffusion time considered is $\tau_{m} \approx$ $30 \mu \mathrm{s}$, which is much larger than observed $\left(\tau_{m} \approx 1 \mu \mathrm{s}\right)$.

Transverse components of $\boldsymbol{B}$ were also measured. Figure 5 shows a time series of the $B_{y}$ component of the magnetic field in the $x-z$ plane. Large-amplitude magnetosonic waves $\left(B_{y} / B_{0} \sim 0.25\right)$ can be seen propagating away from the target. The waves are associated with the magnetic cavity and dissipate as the cavity collapses at late time.

From our 2D planes of vector magnetic field $\boldsymbol{B}$ and electrostatic potential $\Phi$, we can calculate the current density $\boldsymbol{J}=\nabla \times \boldsymbol{B}$ and electrostatic electric field $\boldsymbol{E}=-\nabla \Phi$, where we take $\mathrm{d} \boldsymbol{E} / \mathrm{d} t \sim 0$ (the electric fields are slowly changing relative to the rate at which signals are sampled). We can also estimate the charge density $\rho=-\epsilon_{0} \nabla^{2} \Phi$. The results are shown in Figures 6 ( $x-z$ plane) and 7 ( $x-y$ plane), both at time $t=0.5 \mu \mathrm{s}$. Like Figure 4, Figure 6(a) shows a large magnetic cavity for $x \lesssim 15 \mathrm{~cm}$. Outside of the cavity, the magnetic compression is dominantly directed along $+\hat{z}$, which is the direction of the background field $B_{0}$. At the same time, Figure 6(c) shows that the transition from $B_{z}=0$ in the cavity to $B_{z}>B_{0}$ outside the cavity is associated with a large, negative (into-the-plane) current density $J_{y} \approx$ $-200 \mathrm{~A} \cdot \mathrm{cm}^{-2}$. A smaller amplitude current density $\left(J_{y} \approx\right.$ $30 \mathrm{~A} \cdot \mathrm{cm}^{-2}$ ) is associated with the leading edge of the magnetic compression. The compression is also associated with an outward radially directed electric field of magnitude $|\boldsymbol{E}| \approx 100 \mathrm{~V} \cdot \mathrm{cm}^{-1}$, which reverses sign near the peak of the compression and grows to $|\boldsymbol{E}| \approx 300 \mathrm{~V} \cdot \mathrm{cm}^{-1}$ near the cavity edge, as shown in Figure 6(b). The corresponding charge density $\rho$ is shown in Figure 6(d), which indicates that there is a net positive charge coincident with the magnetic compression. Lineouts at $z=0$ from each of these quantities are shown in Figure 6(e).

These features are also reproduced in the $x-y$ plane (Figure 7). Additionally, Figure 7(c) clearly shows the directionality of the diamagnetic current that supports the 

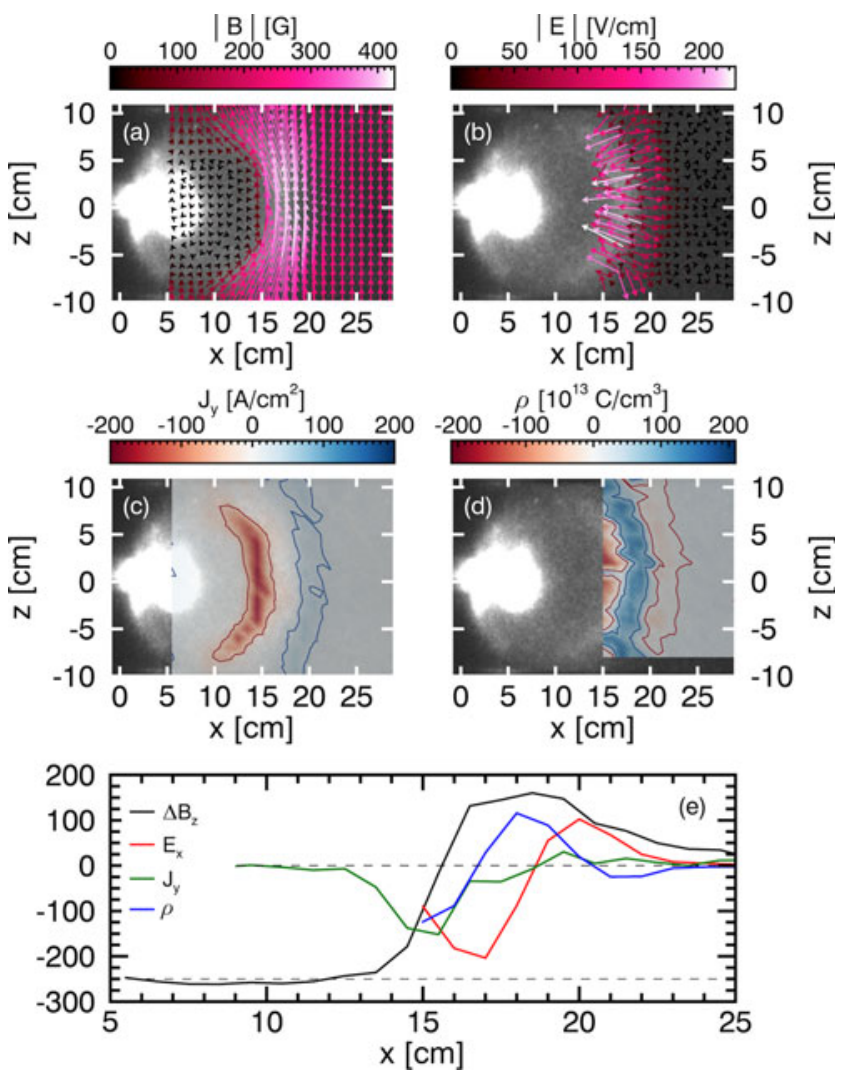

Figure 6. Measured and derived quantities in the $x-z$ plane at time $t=$ $0.5 \mu$ s. (a) Measured vector magnetic field $\boldsymbol{B}$. (b) Vector electric field $\boldsymbol{E}$ derived from the gradient of the measured electrostatic potential. (c) $Y$ component of the current density $J_{y}$, derived from the measured magnetic field. (d) Charge density derived from the measured potential. (e) Profiles taken along $z=0$ in (a)-(d). Also shown in (a)-(d) is an image of plasma self-emission at the same time.

magnetic cavity. Together, the features in Figures 6 and 7 are consistent with a simple model of the piston-ambient plasma interaction. An initial radial inwardly directed ambipolar electric field is created between the relatively unmagnetized laser-plasma ions and magnetized electrons. This field drives $\boldsymbol{E} \times \boldsymbol{B}$ drifts of the electrons, which establishes a clockwise azimuthal diamagnetic current. The current is also supported by electron pressure gradient drifts $(\nabla P \times \boldsymbol{B})$ inherent to the laser-plasma profile. Concurrently, the pile-up of ions (relative to the ambient plasma) at the leading edge creates an outwardly directed electric field, which drives an oppositely directed azimuthal current that acts to increase (compress) the magnetic field.

\section{Discussion and conclusions}

The results of Section 3 show that highly repeatable, detailed and volumetric electrostatic potential and magnetic field datasets can be acquired by combining a high-repetitionrate laser and the LAPD. This in turn allows corresponding quantities of the electrostatic electric field, charge density
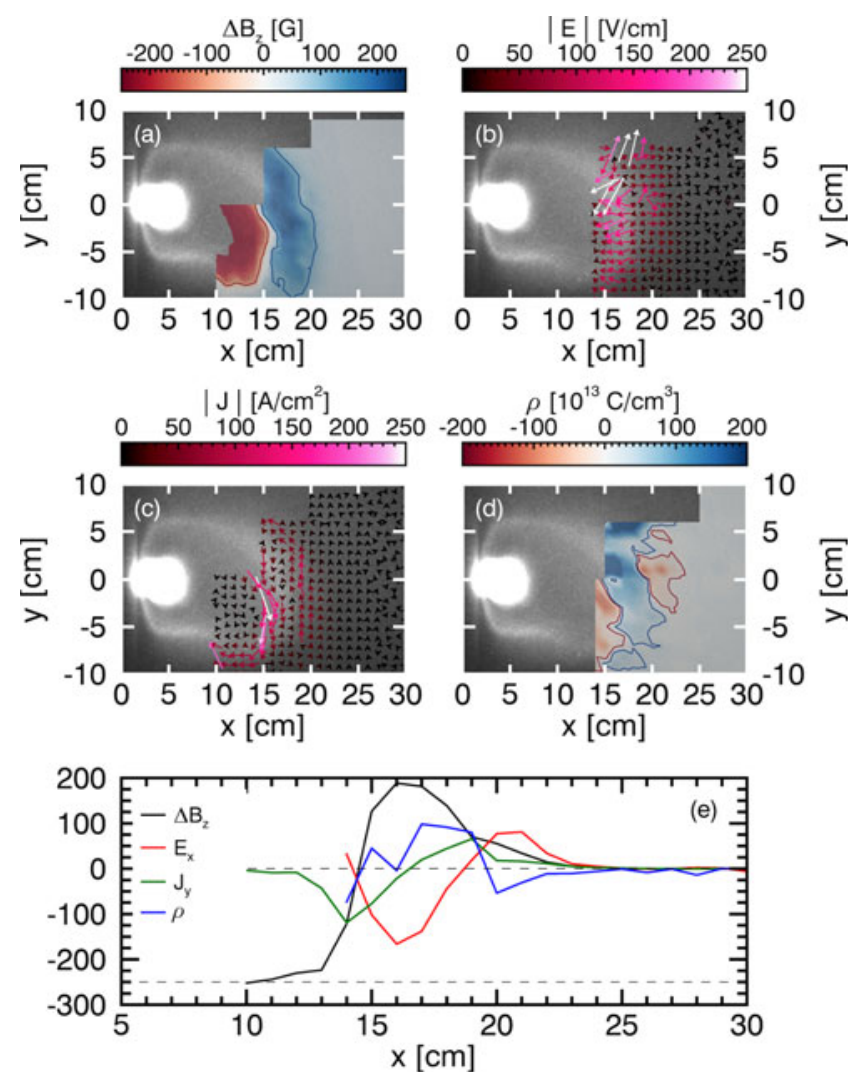

Figure 7. Measured and derived quantities in the $x-y$ plane at time $t=0.5 \mu \mathrm{s}$. (a) $Z$-component of the measured relative magnetic field $\Delta B_{z}=B_{z}-B_{0}$. (b) Vector electric field $\boldsymbol{E}$ derived from the gradient of the measured electrostatic potential. (c) Vector current density $\boldsymbol{J}$, derived from the measured magnetic field. (d) Charge density derived from the measured potential. (e) Profiles taken along $y=0$ in (a)-(d). Also shown in (a)-(d) is an image of plasma self-emission at the same time.

and current density to also be calculated. Additionally, localized, volumetric measurements of the plasma density and temperature can be acquired with Langmuir probes or Thomson scattering, both of which have previously been fielded on the LAPD for laser experiments ${ }^{[22,23]}$. Largescale structure or charge-state evolution of laser-plasma or ambient ions through self-emission can also be imaged with high-repetition, filtered fast-gate cameras ${ }^{[20]}$. Finally, the measurements shown here were confined to the immediate vicinity of the target, but they can be easily extended to regions farther downstream to study, for example, propagating waves.

This high-repetition platform thus provides a rich testbed for exploring the interaction between super-Alfvénic laser plasmas and magnetized ambient plasmas relevant to laboratory astrophysics. Indeed, the experiments presented here were modeled after previous low-repetition experiments on the LAPD that explored perpendicular magnetized collisionless shocks ${ }^{[24,25]}$. In this setup, the laser plasma acts as a supersonic piston to sweep up and accelerate ambient ions until they form a shock ${ }^{[26]}$. While the higher-energy 
(though similar intensity) laser used in previous experiments allows for more energetic pistons, a high-repetition-rate laser allows for a more efficient exploration of the parameter space (background density and magnetic field, ambient gas fill, piston speed, etc.) for shock physics.

Other applications of this platform are currently being pursued. Fast-gate imaging of the laser plasma shows the formation and evolution of flute-like, Rayleigh-Taylor or large-Larmor-radius magnetic instabilities ${ }^{[20,27]}$. Future work will utilize thousands of shots to correlate fluctuations in the magnetic and electric fields and determine the instability growth rate ${ }^{[28]}$. Kinetic-scale magnetospheres are also possible to explore by coupling the laser plasma with an ion-scale $(\sim 10 \mathrm{~cm})$ magnetic dipole field ${ }^{[29]}$. Of particular interest is the dynamics and 3D structure of the interaction between a super-Alfvénic flow and the dipole field, which can be mapped out at high resolution over thousands of shots. Finally, the large axial size of the LAPD, combined with the high-repetition-rate laser, provides a unique opportunity to study the waves and instabilities associated with parallel magnetized collisionless shocks. Here, the laserplasma piston streams along the background field and excites electromagnetic ion beam instabilities, which can be scanned over multiple planes along the length of the machine. Such experiments are currently ongoing ${ }^{[30]}$.

\section{Acknowledgements}

This work was supported by the DTRA under Contract No. HDTRA1-12-1-0024, and the DOE under Contract Nos. DESC0006538 and DE-SC0017900. Thanks to Brent Dane (MIC) for helping to commission the Peening laser system, and Andi Henning for facilitating the loan of the Peening laser from the Space and Naval Warfare Systems Command. Experiments were performed at the UCLA Basic Plasma Science Facility (BaPSF), supported by the DOE and NSF.

\section{References}

1. B. A. Remington, R. P. Drake, and D. D. Ryutov, Rev. Mod. Phys. 78, 755 (2006).

2. G. Gregori, B. Reville, and F. Miniati, Phys. Rep. 601, 1 (2015).

3. B. A. Remington, D. Arnett, R. Paul Drake, and H. Takabe, Science 284, 1488 (1999).

4. D. D. Ryutov, B. A. Remington, H. F. Robey, and R. P. Drake, Phys. Plasmas 8, 1804 (2001).

5. D. B. Schaeffer, W. Fox, D. Haberberger, G. Fiksel, A. Bhattacharjee, D. H. Barnak, S. X. Hu, and K. Germaschewski, Phys. Rev. Lett. 119, 025001 (2017).

6. G. Fiksel, W. Fox, A. Bhattacharjee, D. H. Barnak, P.-Y Chang, K. Germaschewski, S. X. Hu, and P. M. Nilson, Phys. Rev. Lett. 113, 105003 (2014).

7. I. F. Shaikhislamov, Y. P. Zakharov, V. G. Posukh, A. V. Melekhov, V. M. Antonov, E. L. Boyarintsev, and A. G. Ponomarenko, Plasma Phys. Control. Fusion 56, 125007 (2014).
8. G. Gregori, A. Ravasio, C. D. Murphy, K. Schaar, A. Baird, A. R. Bell, A. Benuzzi-Mounaix, R. Bingham, C. Constantin, R. P. Drake, M. Edwards, E. T. Everson, C. D. Gregory, Y. Kuramitsu, W. Lau, J. Mithen, C. Niemann, H. Park, B. A. Remington, B. Reville, A. P. L. Robinson, D. D. Ryutov, Y. Sakawa, S. Yang, N. C. Woolsey, M. Koenig, and F. Miniati, Nature 481, 480 (2012).

9. M. VanZeeland, W. Gekelman, S. Vincena, and G. Dimonte, Phys. Rev. Lett. 87, 105001 (2001).

10. W. Gekelman, M. Van Zeeland, S. Vincena, and P. Pribyl, J. Geophys. Res. 108, 1281 (2003).

11. W. Gekelman, A. Collette, and S. Vincena, Phys. Plasmas 14, 062109 (2007).

12. A. Collette and W. Gekelman, Phys. Rev. Lett. 105, 195003 (2010).

13. J. Bonde, S. Vincena, and W. Gekelman, Phys. Rev. E 92, 051102 (2015).

14. C. Niemann, C. G. Constantin, D. B. Schaeffer, A. Tauschwitz, T. Weiland, Z. Lucky, W. Gekelman, E. T. Everson, and D. Winske, J. Instrum. 7, P03010 (2012).

15. W. Gekelman, P. Pribyl, Z. Lucky, M. Drandell, D. Leneman, J. Maggs, S. Vincena, B. Van Compernolle, S. K. P. Tripathi, G. Morales, T. A. Carter, Y. Wang, and T. DeHaas, Rev. Sci. Instrum. 87, 025105 (2016).

16. C. Dane, L. Zapata, W. Neuman, M. Norton, and L. Hackel, IEEE J. Quantum Electron. 31, 148 (1995).

17. C. B. Dane and L. A. Hackel, High-Pulse-Energy Phase Conjugated Laser System, Phase Conjugate Laser Optics, (2003).

18. E. T. Everson, P. Pribyl, C. G. Constantin, A. Zylstra, D. Schaeffer, N. L. Kugland, and C. Niemann, Rev. Sci. Instrum. 80, 113505 (2009).

19. M. J. Martin, J. Bonde, W. Gekelman, and P. Pribyl, Rev. Sci. Instrum. 86, 053507 (2015).

20. P. Heuer, D. Schaeffer, E. Knall, C. Constantin, L. Hofer, S. Vincena, S. Tripathi, and C. Niemann, High Energy Density Phys. 22, 17 (2017).

21. D. B. Schaeffer, A. S. Bondarenko, E. T. Everson, S. E. Clark, C. G. Constantin, and C. Niemann, J. Appl. Phys. 120, 043301 (2016).

22. C. Niemann, W. Gekelman, C. G. Constantin, E. T. Everson, D. B. Schaeffer, S. E. Clark, D. Winske, A. B. Zylstra, P. Pribyl, S. K. P. Tripathi, D. Larson, S. H. Glenzer, and A. S. Bondarenko, Phys. Plasmas 20, 012108 (2013).

23. D. B. Schaeffer, C. G. Constantin, A. S. Bondarenko, E. T. Everson, and C. Niemann, Rev. Sci. Instrum. 87, 11E701 (2016).

24. C. Niemann, W. Gekelman, C. G. Constantin, E. T. Everson, D. B. Schaeffer, A. S. Bondarenko, S. E. Clark, D. Winske, S. Vincena, B. Van Compernolle, and P. Pribyl, Geophys. Res. Lett. 41, 7413 (2014).

25. D. B. Schaeffer, D. Winske, D. J. Larson, M. M. Cowee, C. G. Constantin, A. S. Bondarenko, S. E. Clark, and C. Niemann, Phys. Plasmas 24, 041405 (2017).

26. A. S. Bondarenko, D. B. Schaeffer, E. T. Everson, S. E. Clark, B. R. Lee, C. G. Constantin, S. Vincena, B. Van Compernolle, S. K. P. Tripathi, D. Winske, and C. Niemann, Nature Phys. 13, $573(2017)$.

27. J. D. Huba, J. G. Lyon, and A. B. Hassam, Phys. Rev. Lett. 59, 2971 (1987).

28. A. Collette and W. Gekelman, Phys. Plasmas 18, 055705 (2011).

29. F. Cruz, E. P. Alves, R. A. Bamford, R. Bingham, R. A. Fonseca, and L. O. Silva, Phys. Plasmas 24, 022901 (2017).

30. P. V. Heuer, M. S. Weidl, R. Dorst, D. B. Schaeffer, A. S. Bondarenko, S. Tripathi, B. Van Compernolle, S. Vincena, C. G. Constantin, and C. Niemann, Phys. Plasmas, in press (2018). 